\title{
O USO DA ACUPRESSÃO PARA EVOLUÇÃO DO TRABALHO DE PARTO E ALÍVIO DA DOR
}

\author{
Reginaldo Roque Mafetoni ${ }^{1}$, Antonieta Keiko Kakuda Shimo ${ }^{2}$
}

\begin{abstract}
RESUMO: Esta revisão integrativa teve o objetivo de buscar as evidências disponíveis na literatura que abordassem o uso da acupressão durante a evolução do trabalho de parto e alívio da dor. Realizou-se uma pesquisa nas bases de dados Scopus, Medline, Cinahal e SciELO, viabilizando a inclusão de 10 estudos, publicados entre 2002 e 2012, que aplicaram a acupressão nos pontos Sanyinyiao, Hegu e Zhiyin durante a fase ativa do trabalho de parto. Os resultados demonstraram que a técnica em análise aparenta diminuir os escores de dor e encurtar o tempo da primeira fase do trabalho de parto. Dois dos estudos consultados apresentaram taxas inferiores de cesarianas nos grupos que receberam a invertenção. A acupressão pode figurar como alternativa não invasiva a ser utilizada por enfermeiros e outros profissinais treinados como meio de obter melhora na qualidade do atendimento à parturientes.

DESCRITORES: Acupressão; Dor do parto; Trabalho de parto.

\section{THE USE OF ACUPRESSURE FOR THE PROGRESSION OF LABOR IN CHILDBIRTH AND FOR PAIN RELIEF}

ABSTRACT: This integrative review aimed to seek evidence available in the literature addressing the use of acupressure during the progression of labor and for pain relief. Research was undertaken in the databases Scopus, Medline, Cinahl and SciELO, including 10 studies, published between 2002 and 2012, which applied acupressure on the Sanyinyiao, Hegu and Zhiyin points during the active phase of labor. The results showed that the technique under analysis reduces the pain scores and shortens the duration of the first phase of labor. Two of the studies consulted showed reduced rates of caesareans in the groups which received the intervention. Acupressure can figure as a non-invasive alternative to be used by nurses and other trained professionals as a means of obtaining improvements in the quality of care given to parturient women.

DESCRIPTORS: Acupressure; Labor pain; Labor obstetric.

\section{EL USO DE LA ACUPRESIÓN PARA EVOLUCIÓN DEL TRABAJO DE PARTO Y ALIVIO DEL DOLOR}

RESUMEN: Esta revisión integrativa tuvo el objetivo de buscar evidencias disponibles en la literatura que hablan acerca del uso de la acupresión durante la evolución del parto y alivio del dolor. Fue realizada una investigación en las bases de datos Scopus, Medline, Cinahal y SciELO, viabilizando la inclusión de 10 estudios, publicados entre 2002 y 2012, que aplicaron la acupresión en los puntos Sanyinyiao, Hegu y Zhiyin durante la fase activa del trabajo de parto. Los resultados muestran que la técnica en análisis parece disminuir los niveles de dolor y acortar el tiempo de la primera fase del trabajo de parto. Dos de los estudios consultados presentaron tasas inferiores de cesáreas en los grupos en que fue hecha la intervención. La acupresión puede ser una alternativa no invasiva a ser utilizada por enfermeros y otros profesionales aptos como medio de obtener mejoría en la cualidad del atendimiento a las parturientes.

DESCRIPTORES: Acupresión; Dolor del parto; Trabajo de parto.

\footnotetext{
${ }^{1}$ Enfermeiro. Mestrando pelo Programa de Pós-Graduação em Enfermagem da Faculdade de Ciências Médicas da Universidade Estadual de Campinas-UNICAMP.

${ }^{2}$ Enfermeira. Doutora em Enfermagem. Professora do Curso de Graduação e do Programa de Pós-Graduação da Faculdade de Ciências Médicas da UNICAMP.
} 


\section{INTRODUÇÃO}

A dor que a gestante sente durante a evolução do Trabalho de Parto (TP) e parto é única para cada mulher em função dos diferentes fatores que a influenciam. Dentre estes fatores incluem-se a cultura, a história familiar, a ansiedade, o medo, a experiência anterior de parto, a preparação para o parto e o suporte oferecido durante este processo. Uma vez diagnosticado o TP e a regularidade das contrações a dor pode ser aliviada, principalmente, nas ocorrências em que incide sobre um organismo materno com patologia associada ${ }^{(1)}$.

Na Medicina Tradicional Chinesa (MTC), vários métodos são utilizados de forma eficaz não só para facilitar o trabalho, mas também para gerenciar a dor do parto. O princípio da MTC consiste em equilibrar e harmonizar na pessoa duas entidades opostas de energia, a "negativa" (yin) e a "positiva" (yang), a fim de manter a estabilidade de cada uma por meio, principalmente, da acupuntura, da acupressão, do uso de ervas, entre outras práticas ${ }^{(2)}$.

A MTC parte do princípio de que existem vários canais de energia correndo pelo corpo - os chamados meridianos ou canais - que são alvo de estímulos em determinados pontos (tsubôs ou acupontos), o que permite o reequilíbrio de seu fluxo de energia (chamado de Ki ou Qi em chinês) e de seus órgãos. A dor do parto é vista, assim, como uma consequência do desequilíbrio entre as duas entidades de energia ${ }^{(2)}$.

A técnica de acupressão é uma variação da acupuntura que envolve a pressão com um dos dedos de forma leve ou de média intensidade, aplicando uma massagem circular na pele ou manter-se firme e constante em pontos específicos, em sua maioria bilaterais, sem o uso de agulhas ${ }^{(3)}$. A acupressão visa, principalmente, aliviar o sofrimento mediante a tonificação do Qi fraco, a sedação do Qi superativo ou a dispersão do Qi bloqueado, usando para tanto os tsubôs clássicos a fim de equilibrar e harmonizar as energias internas, mantendo a estabilidade da mente e do corpo ${ }^{(4)}$.

Com base nesses conceitos e procurando somar esforços para a melhoria da assistência à parturiente, propôs-se uma investigação com o objetivo de buscar as evidências científicas disponíveis na literatura que abordem o uso da acupressão para melhora/evolução do TP e alívio da dor.

\section{MÉTODO}

A pesquisa é do tipo revisão integrativa(5), a qual tem a finalidade de reunir e sintetizar resultados de diferentes pesquisas sobre um delimitado tema, de forma sistemática e ordenada. Dessa maneira deu-se a formulação da seguinte questão norteadora: "Quais são as evidências científicas do uso da acupressão para evolução do TP e alívio da dor?”

O levantamento bibliográfico foi realizado acessando as bases eletrônicas: Scopus, Medline via Pubmed, Cinahal e SciELO. A busca dos artigos foi realizada via Biblioteca Eletrônica em uma universidade do Estado de São Paulo, por meio do modo "with full text". Utilizaram-se os descritores do DeCS/MeSH e o operador booliano and em três idiomas distintos (português, inglês e espanhol), resultando nas seguintes combinações: "acupressão and dor do parto" e "acupressão and trabalho de parto".

Foram incluídos artigos originais sem restrição de idiomas, publicados no período de julho de 2002 a julho de 2012, que descreviam o uso da acupressão para alívio da dor no TP a partir da leitura de seus títulos e resumos. Excluíram-se os artigos de revisão.

A coleta de informações deu-se através do preenchimento de um instrumento elaborado pelos pesquisadores e aplicado a cada artigo da amostra final desta revisão. O instrumento apresentou as seguintes informações: registro do título, ano, revista, base de dados, autores e identificação profissional, país de origem, objetivos, método, características da amostra, resultados, conclusão e nível de evidência científica.

A apresentação dos resultados e discussão geral do uso da acupressão durante a evolução do TP foi feita de forma descritiva, possibilitando ao leitor a avaliação da aplicabilidade da revisão integrativa elaborada de forma a atingir o objetivo deste estudo.

\section{RESULTADOS}

A busca bibliográfica realizou-se em agosto de 2012, sendo a amostra final desta revisão integrativa constituída por 10 artigos (Tabela 1).

Na tabela 2 são caracterizados os estudos inclusos nesta revisão. Todos foram classificados com nível de evidência II, por serem procedentes de Ensaios Controlados e Randomizados (ECRs) ${ }^{(6)}$.

Foram identificados cinco ECRs que avaliaram o uso da acupressão no ponto BP6 (Sanyinyiao) $)^{(2,8,11)}$; um estudou o ponto B67 (Zhiyin) e IG4 (Hegu) na mesma amostra $^{(7)}$; três utilizaram o ponto IG4 ${ }^{(12,14-15)}$; outro estudo, os dois pontos: BP6 e IG4 ${ }^{(13)}$, todos bilateriais. Destes, 8 acrescentaram o Grupo de Toque (GT) (2,8-14), realizando um contato superficial no mesmo ponto em forma de simulação da acupressão; um aplicou a 
Tabela 1 - Seleção dos artigos de pesquisa nas bases de dados SCOPUS, MEDLINE, CINAHL e SciELO de acordo com os critérios de inclusão estabelecidos. Brasil, 2012

\begin{tabular}{lccccc}
\hline & Scopus & Medline & Cinahl & SciELO & Total \\
\hline Produção Encontrada & 41 & 19 & 17 & 0 & 77 \\
Não responde a questão da pesquisa & 13 & 9 & 10 & 0 & 32 \\
Não disponível & 7 & 0 & 2 & 0 & 9 \\
Revisões & 14 & 2 & 3 & 0 & 19 \\
Repetido & 0 & 5 & 2 & 0 & 7 \\
Total selecionado & $\mathbf{7}$ & $\mathbf{3}$ & $\mathbf{0}$ & $\mathbf{0}$ & $\mathbf{1 0}$ \\
\hline
\end{tabular}

Tabela 2 - Distribuição dos artigos selecionados segundo autores, revista, ano de publicação, base de dados, profissional que aplicou e o país de origem. Brasil, 2012

\begin{tabular}{|c|c|c|c|c|c|}
\hline Autores & Revista & Ano & Banco de dados & Quem Aplicou & Local do estudo \\
\hline $\mathrm{Lee}^{(2)}$ & $\begin{array}{l}\text { Taehan Kanho Hakhoe } \\
\text { Chi }\end{array}$ & 2003 & Medline & Enfermeiro & Coreia do Sul \\
\hline Chung et al. ${ }^{(7)}$ & J Nurs Res & 2003 & Medline & Enfermeiro & Taiwan \\
\hline Lee et al. ${ }^{(8)}$ & $\begin{array}{l}\text { J Altern Complement } \\
\text { Med }\end{array}$ & 2004 & Scopus & Enfermeiro & Coreia do Sul \\
\hline Chang et al. ${ }^{(9)}$ & $\begin{array}{l}\text { Taehan Kanho Hakhoe } \\
\text { Chi }\end{array}$ & 2004 & Medline & Enfermeiro & Coreia do Sul \\
\hline Kashanian et al. ${ }^{(10)}$ & $\begin{array}{l}\text { J Matern Fetal Neonatal } \\
\text { Med. }\end{array}$ & 2010 & Scopus & Obstetra & Irã \\
\hline Hjelmstedt et al.(11) & $\begin{array}{l}\text { Acta Obst et Ginecol } \\
\text { Scand. }\end{array}$ & 2010 & Scopus & Multiprofissional & Índia \\
\hline Kordi et al..$^{(12)}$ & Hayat & 2010 & Scopus & Enfermeiro & Irã \\
\hline Salehian et al. ${ }^{(13)}$ & SJKU & 2011 & Scopus & Enfermeiro & Irã \\
\hline Hamidzadeh et al.(14) & J Koomesh & 2011 & Scopus & Obstetra & Irã \\
\hline Hajiamini et al. ${ }^{(15)}$ & $\begin{array}{l}\text { Complement Therapies } \\
\text { in Clin Pract }\end{array}$ & 2012 & Scopus & Enfermeiro & Irã \\
\hline
\end{tabular}

técnica de effleurage (deslizamento) ${ }^{(7)}$; outro, pressão com gelo ${ }^{(15)}$ para comparação. Em seis ECRs houve o terceiro grupo, o Grupo Controle (GC) $)^{(7,9,11-13,15)}$.

A mensuração da dor foi a variável predominante dos estudos selecionados ${ }^{(2,7-8,10-15)}$. Destes, cinco avaliaram a duração da primeira etapa do TP ou o tempo para o nascimento em minutos ${ }^{(2,7-8,10,13)}$; um calculou esta variável em horas ${ }^{(7)}$ e somente um ensaio focou no desfecho da via de parto ocorrido em sua amostra ${ }^{(9)}$. Todos os ECRs que mensuraram os níveis dor utilizaram como instrumento a Escala Analógica e Visual (EAV) graduada de 0 a 10, exceto um que trabalhou com escala diferenciada de 0 a $100^{(12)}$.

Os estudos utilizaram como critérios de inclusão gestantes sem comorbidades, feto com boas condições de vitalidade e colo uterino dilatado de 3 a $5 \mathrm{~cm}$, durante a fase ativa do TP, com exceção de um estudo que incluiu parturientes com dilatação a partir de $2 \mathrm{~cm}^{(7)}$. $\mathrm{O}$ tempo de intervenção também sofreu variações, sendo de 30 minutos em cinco estudos ${ }^{(2,8-11)}, 20$ minutos em três ${ }^{(7,13-14)}, 40$ minutos em um estudo ${ }^{(12)}$ e 10 minutos em outro ${ }^{(15)}$.

Com relação aos três ECRs realizados na Coreia do Sul ${ }^{(2,8-9)}$, participaram de um dos estudos ${ }^{(2)} 29$ parturientes no grupo BP6, e 29 no GT. Os escores de dor foram significativamente diferentes entre os dois grupos (pré-intervenção BP6 5.96 \pm 1.90 x GT 6.16 \pm 2.48 , $\mathrm{p}=0,344$ / pós BP6 6.45 $\pm 1.88 \times$ GT 7.48 \pm 1.92 , $\mathrm{p}=0,020$ ), sendo o tempo médio de nascimento menor no grupo BP6 $(145.35 \pm 66,9)$ x GT $(197.77 \pm 93.91) \mathrm{p}=0,036$.

O segundo ensaio oriundo deste país ${ }^{(8)}$ utilizou amostra maior (75 mulheres em TP). Os escores de dor mensurados apresentaram diferenças significativas entre os grupos em todos os momentos após a inter-


= 0,012), com 30 minutos (BP6 7.0 $\pm 1.8 \times$ GT 8.3 \pm 1.8 , p 
$=0,021)$ e com 60 minutos (BP6 7.7 \pm 1.5 x GT 8.9 \pm 1.7 , $\mathrm{p}=0,012)$. $\mathrm{O}$ tempo médio ( $3 \mathrm{~cm}$ de dilatação para o parto) foi menor no grupo BP6 (138.6 \pm 62.0$)$ quando comparado ao GT $(191.2 \pm 83.7) \mathrm{p}=0,006$.

Outro ECR ${ }^{(9)}$ sul-coreano avaliou o índice de cesariana ocorrido em sua amostra $(\mathrm{n}=209)$. O resultado apontou taxas de cesarianas superiores nos GT (29,8\%), GC $(22,4 \%)$ e porcentagens menores de cesarianas no grupo BP6 (12,8\%), demonstrando diferença entre os grupos $(\mathrm{p}=0,049)$ e número de cesáreas significativamente diferentes $(\mathrm{p}=0,035)$.

$\mathrm{Um} \mathrm{ECR}^{(7)}$ realizado em Taiwan utilizou 127 parturientes distribuídas em três grupos. Houve diferença no alívio da dor apontada somente na fase ativa do TP entre os grupos (IG4/B67 0.17 x effleurage 0.66 x GC $0.81, \mathrm{p}=0,041)$, não sendo significativo na fase latente $(\mathrm{p}=0,051)$ e de transição $(\mathrm{p}=0,480)$. A análise para a fase ativa indicou que não houve diferença na diminuição da dor entre os grupos IG4/B67 e effleurage (p = 0,109), mostrando-se relevante somente entre IG4/B67 e GC ( $p=0,017)$. A média de duração da primeira etapa do TP foi menor no grupo IG4/B67 (6.33 \pm 2.55$)$, seguido pelo grupo effleurage (7.13 \pm 3.14$)$ e GC ( $8.45 \pm 4.39)$ $\mathrm{p}=0,019$.

Cinco estudos foram oriundos do Irã ${ }^{(10,12-15)}$. A acupressão no ponto BP6 foi realizada em um ensaio com 60 participantes ${ }^{(10)}$, a intensidade de dor e um exame pélvico foi executado duas horas após a intervenção para comparar os resultados entre os grupos. O tempo médio de duração da fase ativa apresentou-se menor no grupo BP6 $(252.37 \pm 108.5)$ x GT $(441.38 \pm 155.88)$ $\mathrm{p}=0,0001$, o número de cesariana foi maior no GT $(41,7 \%)$ x BP6 (10\%) p=0,0001, com diferença significativa no uso de oxitocina entre os grupos BP6 (41,7\%) x GT $(63,3 \%) p=0,017$. Os escores de dor mensurados foram menores no grupo BP6 quando comparado ao GT (5.87 $\pm 1.77 \times 6.79 \pm 1.52, \mathrm{p}=0,003)$.

A avaliação da acupressão do ponto IG4 foi foco em dois ensaios iranianos ${ }^{(12,14)}$. Os resultados em ambos os estudos foram significativos quando comparados às respostas sobre o alívio da dor. Em um estudo ${ }^{(12)} \mathrm{com}$ 83 participantes apresentou melhoras imediatamente


x GC $41.82 \pm 20.5, p=0,026)$. O outro ${ }^{(14)}$, com 50 parturientes em cada grupo, apresentou as mesmas características em todas as avaliações: após interven-

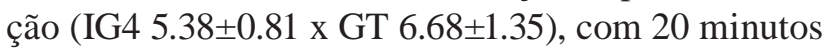
(IG4 6.5 \pm 0.89 x GT 8.26 \pm 1.14 ), com 60 minutos (IG4 $7.12 \pm 1.55$ x GT $8.92 \pm 1.98$ ) e com 120 minutos da in-



Somente um ECR, propôs estudar os dois pontos IG4 e BP6 na mesma amostra ${ }^{(13)}$. Os participantes foram divididos em três grupos equivalentes de 30 . O estudo apontou diferenças após a intervenção com 4cm (IG4 2.6 \pm 0.95 x BP6 2.2 \pm 1.41 x GC 4 \pm 1.25 ), com $6 \mathrm{~cm}$ (IG4 4.1 \pm 1.29 x BP6 4.3 \pm 1.15 x GC 6.9 \pm 1.8 ) e com $8 \mathrm{~cm}$ (IG4 7.10 \pm 1.26 x BP6 6.9 \pm 1.65 x GC 8.2 \pm 1.22 ) de dilatação cervical nos três grupos $(p<0,001)$, sendo que com $10 \mathrm{~cm}$ não houve diferença $(\mathrm{p}=0,7)$. A duração da fase ativa foi menor nos grupos de intervenção (IG4 185 \pm 44 x BP6 174 \pm 74 x GC 225 \pm 20 , p=0,001), porém não significativa com $10 \mathrm{~cm}$ de dilatação uterina até o nascimento $(\mathrm{p}=0,7)$.

Outro estudo do Irã ${ }^{(15)}$ comparou os efeitos da acupressão no ponto IG4 fazendo uso da massagem com gelo no mesmo ponto. Nas comparações de intensidade da dor entre as 90 parturientes, o grupo massagem com gelo, apontaram-se diferenças significativas em todas as avaliações (antes $7.47 \pm 1.94$ e após-intervenção $5.73 \pm 1.74,5.90 \pm 1.84,6.77 \pm 1.97, \mathrm{p}<0,001)$. No grupo IG4 sem gelo, houve diferença na intensidade da dor antes, imediatamente e meia hora pós-intervenção $(7.73 \pm 1.41$, $6.70 \pm 1.44,6.87 \pm 1.45, \mathrm{p}<0,001)$, não significativa depois de uma hora (7.73 \pm 1.50$)$. No grupo placebo a única diferença estatistica foi observada pré e imediatamente pós-intervenção $(7.17 \pm 1.66,6.33 \pm 1.72, \mathrm{p}<0,001)$.

Na Índia, um ECR(11) acrescentou o GC, mantendo $o$ atendimento usual da unidade $(\mathrm{n}=70)$ para comparar com o grupo BP6 $(n=71)$ e GT $(n=71)$. Os escores de dor, antes e após a intervenção, foram menores no grupo BP6 e mais perceptíveis imediatamente após o tratamento (BP6 -4.4 x GT 2.0 x GC 4.7, p <0,001).

\section{DISCUSSÃO}

Devido à seleção de artigos originais, houve um predomínio de estudos com evidência II. Em relação à pergunta da pesquisa, pudemos identificar um crescente interesse na clínica obstétrica, principalmente no Oriente Médio e na Ásia, o uso da acupressão para gerenciar diferentes aspectos do TP. Ressalta-se o fato de não terem sido identificados estudos brasileiros nessa temática.

O enfermeiro destaca-se como o profissional predominante na investigação e aplicação da técnica em parturientes (70\% dos artigos selecionados), reforçando o interesse da categoria em promover o uso de estratégias não invasivas. Humanizar a assistência é prioritariamente respeitar o tempo da mulher no processo de parturição; evitar intervenções desnecessárias 
impostas pelas rotinas hospitalares e reconhecer os aspectos culturais próprios da mulher ${ }^{(16)}$.

Os pontos de acupuntura utilizados foram: o BP6 (Sanyinjiao), presente em seis estudos ${ }^{(2,8-11,13)}$ localizado no meridiano do baço-pâncreas, quatro dedos acima da ponta do maléolo interno, parte posterior à frente da tíbia; o B67 (Zhiyin) em um ECR ${ }^{(7)}$ situado no meridiano da bexiga, na margem ungueal do $5^{\circ}$ dedo do pé; e o IG4 (Hegu), localizado no meridiano do intestino grosso, na depressão entre o primeiro e o segundo ossos metacarpais no seu lado radial, em outros cinco estudos $^{(7,12-15)}$. A acupressão sobre esses pontos de acupuntura é creditado estimular a liberação de oxitocina pela glândula pituitária que, por sua vez, estimula as contrações uterinas para melhorar o processo de trabalho de parto ou para gerenciar a dor do parto ${ }^{(17)}$.

De maneira geral, a aplicação da acupressão caracterizou-se nesta revisão durante a primeira etapa do TP, na fase ativa, marcada por contrações fortes, regulares e dilatação do colo uterino $>3 \mathrm{~cm}$. Tais intervenções ocorreram em um único momento, durante um período que variou entre 10 a 40 minutos, com frequência maior em 30 minutos $^{(2,8-11)}$. Um estudo justificou o tempo utilizado com base na média aproximada de 24 minutos do ciclo de circulação do fluxo de energia no meridiano no corpo $^{(8)}$.

Os efeitos da acupressão sobre a dor durante o TP foi avaliado em 90\% dos ECRs desta revisão integrativa $^{(2,7-8,10-15)}$. Os resultados mostraram-se significativos antes e imeditamente após a intervenção na maioria dos estudos, evidenciado por escores de dor através da EAV menores nos grupos que receberam acupressão real. Somente um estudo ${ }^{(7)}$ relatou não ter encontrado resultados favoráveis nas primeiras avaliações, sendo o único a ter iniciado a intervenção com $2 \mathrm{~cm}$ de dilatação cervical (na fase latente do TP).

Os escores de dor mensurados após 20 minutos ou em um segundo momento demonstraram ponturações proporcionalmente inferiores nos grupos de acupressão em relação aos controles e toques (simulação) em cinco estudos $^{(7-8,13-15)}$. A fase de transição do TP, ou quando o colo uterino atingia $>8 \mathrm{~cm}$, destacou-se como um momento ainda pouco estudado nesta temática. Dois estudos $^{(7,13)}$ avaliaram o nível de dor nesta etapa do TP, porém não encontraram diferenças significativas em suas amostras.

A acupressão nos pontos IG4 e B67 foi comparada à técnica de effleurage (deslizando as mãos nos braços da paciente de cima para baixo durante 10 minutos em cada membro) ${ }^{(7)}$. Na análise durante a fase ativa não houve diferença significativa na diminuição da dor relatada entre os grupos acupressão e effleurage ou entre effleurage e controle, porém foi significativa a diferença da técnica de acupressão comparada ao GC. A comparação da técnica de acupressão ao uso de bola de gelo ( 2 cm de diâmetro) no mesmo ponto, demonstrou escores de dor inferiores após a intervenção em todas as avaliações realizadas no grupo massagem com gelo ${ }^{(15)}$. No grupo acupressão sem gelo, tal relato ocorreu somente em dois momentos após a intervenção, porém esta análise realizou a acupressão com bola de vidro, e não o contato com os dedos indicado para técnica.

O tempo de TP até o nascimento foi mensurado em três estudos ${ }^{(2,8,13)}$, tendo dois deles apontado o tempo para nascimento menor nos grupos de acupressão ${ }^{(2,8)}$ e o terceiro ${ }^{(13)}$, focado na observação a partir de $10 \mathrm{~cm}$ de dilatação cervical até o nascimento, não encontrou diferença entre os grupos. Já o tempo de dilatação total foi calculado em três $\operatorname{ECRs}^{(7,10,13)}$, todos descreveram período inferior desta fase do TP nos grupos de acupressão real.

Não foi apresentado em alguns estudos, ou não ficou claro, o uso de medicamentos para a indução do TP. Apenas um ensaio mencionou não ter ocorrido aumento de oxitocina adicionais ${ }^{(8)}$ e outro citou o uso de oxitocina em maior porcentagem no $\mathrm{GT}^{(10)}$.

A via de parto ocorrida em dois estudos ${ }^{(9-10)}$ evidenciou taxas de cesarianas inferiores nos grupos que receberam a acupressão em relação aos demais grupos. Em um ECR ${ }^{(10)}$ o número de cesáreas foi aproxidamente três vezes maior no grupo de simulação da técnica.

A acupressão não foi associada a riscos de infecções, uma vez que a pele não é penetrada por agulhas e na maioria dos estudos não há sequer custos com equipamentos. Parece ser um método simples, seguro e de baixo custo. A maioria dos estudos selecionados nesta revisão aplicou a acupressão para alívio da dor durante o TP, não havendo descrições de efeitos colaterais para a mãe ou recém-nascido(RN).

\section{CONSIDERAÇÕES FINAIS}

Os artigos inclusos nesta revisão integrativa foram classificados com evidência forte (II). Três pontos de acupuntura foram utilizados para a acupressão durante o TP: BP6 (Sanyinyiao), IG4 (Hegu) e B67 (Zhiyin), sendo mais frequentes os dois primeiros.

A fase ativa do TP foi o período que predominou a aplicação da acupressão. Os níveis de dor mensurados 
pela EAV nesta fase foram significativos nas primeiras avaliações em todos os estudos. Em uma segunda avaliação, as pontuações da dor foram menores em aproximadamente metade dos ensaios. Na fase de transição do TP foram feitas poucas avaliações, não havendo evidências significativas.

O tempo do TP ou para o nascimento do RN foi a segunda variável mais pesquisada. O resultado desta revisão apontou que o uso da acupressão parece diminuir este período, encurtando a primeira etapa do $\mathrm{TP}$, embora um estudo descrevesse não ter encontrado diferença em parturientes com dilatação cervical total até o nascimento.

A via de parto foi pouco explorada, porém houve evidências inferiores de cesarianas nos grupos de receberam acupressão.

Em contextos onde métodos mais eficazes de alívio da dor estão disponíveis, tais como analgesia epidural, a acupressão pode ser uma alternativa não invasiva no início do TP, utilizável por enfermeiros ou outros profissionais treinados a fim de melhorar a qualidade do atendimento às parturientes. No entanto, novos estudos de eficácia, bem como eficiência, são necessários para determinar a aplicabilidade clínica da acupressão na fase avançada do TP.

\section{REFERÊNCIAS}

1. Ministério da Saúde (BR). Parto, aborto e puerpério: assistência humanizada à mulher. Brasília: Ministério da Saúde; 2001.

2. Lee MK. Effects of San-Yin-Jiao (SP6) acupressure on labor pain, delivery time in women during labor. Taehan Kanho Hakhoe Chi. [Internet] 2003;33(6) [acesso em 01 ago 2012]. Disponível: http://www.ncbi.nlm.nih.gov/ pubmed/15314393

3. Ulrich W. Livre-se das dores pela acupuntura e acupressura. Tradução de Maria Madalena Würth Teixeira. Rio de Janeiro: Ediouro; 1980.

4. Jamey C, Mojay G. Shiatsu um guia completo. $3^{\text {a }}$ ed. São Paulo: Pensamento; 2001.

5. Ganong LH. Integrative reviews of nursing research. Res. nurs. health. [Internet] 1987;10(1) [acesso em 01 ago 2012]. Disponível: http://www.ncbi.nlm.nih.gov/ pubmed/3644366

6. Stetler CB, Brunell M, Giuliano KK, Morsi D, Prince L, Newell-Stokes V. Evidence-based practice and the role of nursing leadership. J Nurs Adm. [Internet] 1998;28(78) [acesso em 01 ago 2012]. Disponível: http://www. ncbi.nlm.nih.gov/pubmed/9709696

7. Chung UL, Hung LC, Kuo SC, Kuang CL. Effects of LI4 and BL 67 Acupressure on labor pain and uterine contractions in the first stage of labor. J Nurs Res. [Internet] 2003; 11(4) [acesso em 01 ago 2012]. Disponível: http://www.ncbi.nlm.nih.gov/ pubmed/14685931

8. Lee MK, Chang SB, Kang DH. Effects of SP6 Acupressure on labor pain and length of delivery time in women during labor. J Alt Complem Med. [Internet] 2004;10 (6) [acesso em 02 ago 2012]. Disponível: http://www.birthpartner.com.au/Acupressure_ DVD_ files/Effects\%20 of \%20SP6\%20acupressure\%20 on\%20labor\%20pain\%20and\%20length\%20of\%20 delivery\%20time\%20in\%20women\%20during\%20 labor.pdf

9. Chang SB, Park YW, Cho JS, Lee MK, Lee BC, Lee SJ. Differences of cesarean section rates according to SanYin-Jiao (SP6) acupressure for women in labor. Taehan Kanho Hakhoe Chi. [Internet] 2004;34(2) [acesso em 01 ago 2012]. Disponível: http://www.ncbi.nlm.nih.gov/ pubmed/15314328

10. Kashanian M, Shahali S. Effects of acupressure at the Sanyinjiao point (SP6) on the process of active phase of labor in nulliparas women. J Matern Fetal Neonatal Med. [Internet] 2010;23(7) [acessso em 02 ago 2012]. Disponível: http://medicine.tums.ac.ir:803/files/ article/8228/Effects\%20of\%20acupressure\%20at\%20 the \%20Sanyinjiao\%20point\%20(SP6)\%20on\%20 the\%20process\%20of.pdf

11. Hjelmstedt A, Shenoy ST, Stener-Victorin E, Lekander M, Bhat M, Balakumaran L et al. Acupressure to reduce labor pain: a randomized controlled trial. Acta Obstet Gynecol Scand. [Internet] 2010;89 [acesso em 02 ago 2012]. Disponível: http://onlinelibrary.wiley.com/doi/1 0.3109/00016349.2010.514323/abstract

12. Kordi M, Firoozi M, Esmaili H. Acupressure on labor pain in the first stage of labor in nuliparous women. HAYAT. [Internet] 2010; 16(3-4) [acesso em 02 ago 2012]. Disponível: http://journals.tums.ac.ir/upload_ files/pdf/_17680.pdf

13. Salehian T, Dehcheshmaei FS, Pirak A, Kazemian A, Atarodi Z, Righi SDN. Comparison of the effect of Hoku Point (LI4) acupressure with that of San-Yin-Jiao (SP6) acupressure on labor pain and the length of delivery time in primiparous women. SJKU. [Internet] 2011;16(1) 
[acesso em 02 ago 2012]. Disponível: http://sjku.hbi.ir/ browse.php?a_code=A-10-1-282\&sid=1\&slc_lang=en

14. Hamidzadeh A, Shahpourian F, Orak RJ. Effects of LI4 acupressure on labor pain in the first stage of labor. Koomesh. [Internet] 2011;12(2) [acesso em 02 ago 2012]. Disponível: http://www.sid.ir/fa/VEWSSID/J_ pdf/58313893813.pdf

15. Hajiamini Z, Masoud SN, Ebadi A, Mahboubh A, Matin AA. Comparing the effects of ice massage and acupressure on labor pain reduction. Complement Ther Clin Pract. [Internet] 2012;18(3) [acesso em 02 ago 2012]. Disponível: http://www.ctcpjournal.com/article/ S1744-3881(12)00041-2/fulltext

16. Moreira KAP, Araújo MAM, Fernandes AFC, Braga VAB, Marques JF, Queiroz MVO. O significado do cuidado ao parto na voz de quem cuida: uma perspectiva à luz da humanização. Cogitare enferm. [Internet] 2009;14(4) [acesso em 15 ago 2012]. Disponível: http://ojs.c3sl.ufpr.br/ojs2/index.php/cogitare/article/ view/16389

17. Cook A, Wilcox E. Pressuring pain: alternative therapies for labor pain management. AWHONN lifelines. [Internet] 1997;1(2) [acesso em 15 ago 2012]. Disponível: http://onlinelibrary.wiley.com/ doi/10.1111/j.1552-6356.1997.tb00929.x/abstract 